\title{
Slow Growth Polymerization of Methyl Methacrylate in Toluene by Butyllithium
}

\author{
Koichi Hatada, Mitsuru Furomoto, Tatsuki Kitayama, \\ Yutaka TsUBOKURA, and Heimei YUKI \\ Department of Chemistry, Faculty of Engineering Science, \\ Osaka University, Toyonaka, Osaka 560, Japan.
}

(Received December 7, 1979)

\begin{abstract}
In this paper, a new method of ionic polymerization is described and designated as "slow growth polymerization." An initiator solution was slowly added to a monomer solution to enable it to rest on top as a separate liquid phase and the polymerization was allowed to proceed without stirring. The initiation occurs at the interface between the solutions of initiator and monomer, and the polymerization proceeds from top to bottom. When methyl methacrylate was polymerized by this method, using butyllithium as initiator in toluene, the molecular weight and isotacticity of the polymer formed were much higher than those of the polymer obtained by the ordinary method. The mechanism for the increase in the isotacticity is discussed in some detail.
KEY WORDS Anionic Polymerization without Stirring / Anionic Slow Growth Polymerization / Methyl Methacrylate / Butyllithium / 1,1-Di- phenylhexyllithium / Lithium Methoxide / Isotactic Poly(methyl methacrylate)

In our previous communications, ${ }^{1-3}$ we briefly reported on the polymerization and copolymerization of methyl methacrylate by the slow growth method. Slow growth polymerization is a special method for ionic polymerization, wherein the initiator solution is placed on the monomer solution so as to rest on top of it as a separate liquid phase and the polymerization is allowed to proceed without stirring (Figure 1). In this polymerization, the initiation occurs at the interface between the initiator and monomer solutions, and the polymerization reaction proceeds from top to bottom. By this method we can obtain polymers and copolymers of different tacticity, molecular weight or composition from those obtained by ordinary method. For instance, poly(methyl methacrylate) prepared by slow growth polymerization initiated by butyllithium had a higher isotacticity and molecular weight than the polymer obtained by the ordinary method. ${ }^{1}$

In this paper, slow growth polymerizations of undeuterated and perdeuterated methyl methacrylates by butyllithium were studied in detail in toluene and the number of the initiator fragments in the polymer and oligomer chains was measured according to the method shown in a previous publication. ${ }^{4}$ Diffusion of isotactic and syndiotactic oligomers of methyl methacrylate into the solution of an isotactic polymer was studied by using ${ }^{1} \mathrm{H}$ NMR spectroscopy. On the basis of these results, the

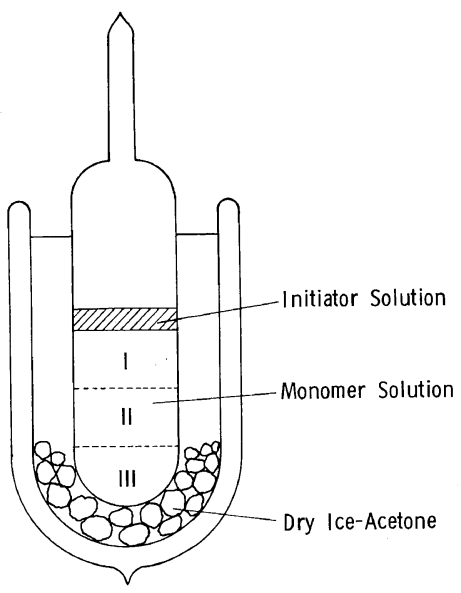

Figure 1. Slow growth polymerization. 
mechanism of slow growth polymerization is discussed in some detail.

\section{EXPERIMENTAL}

Methyl methacrylate was purified by fractional distillation under nitrogen pressure. The monomer thus purified was distilled over calcium dihydride under high vacuum just before use.

Perdeuterated methyl methacrylate was prepared from acetone cyanohydrin- $d_{7}$ and methanol- $d_{4}$ according to the method of Crawford ${ }^{5}$ and purified in the same way as undeuterated methyl methacrylate.

Toluene was dried by adding a small amount of a solution of butyllithium in toluene and was then distilled under high vacuum.

Butyllithium was prepared from 1-chlorobutane and metallic lithium in heptane.

1,1-Diphenylhexyllithium was synthesized by the reaction of equimolar amounts of butyllithium and 1,1-diphenylethylene in toluene.

Slow growth polymerization was carried out in the following way. The monomer solution was cooled to a desired polymerization temperature in the reaction vessel under nitrogen atmosphere and the initiator solution was slowly added on to the monomer solution as a separate liquid phase; and then, the polymerization was allowed to proceed without stirring. After a desired period, the polymerization vessel was cooled in liquid nitrogen and carefully broken. The reaction mass was sliced into three portions, and designated as I, II, and III from top to bottom. Each portion of the reaction mass was put into a large amount of methanol to precipitate the polymer formed. After standing overnight, the precipitated polymer was collected by filtration, washed with methanol and dried in vacuo at room temperature.

The concentration of butyllithium in the initiator layer of the slow growth polymerization was estimated in the following way by Fourier-transform NMR spectroscopy. The spectrometer used was JNM-FX100 (JEOL) and was operated at $100 \mathrm{MHz}$. An aliquot amount $(0.11 \mathrm{ml})$ of $0.9 \mathrm{moll}^{-1}$ solution of methyl methacrylate in toluene- $d_{8}$ was put into a $5 \mathrm{~mm}$ NMR sample tube under nitrogen atmosphere and a $0.12 \mathrm{ml}$ solution of butyllithium in heptane- $d_{16}$ $\left(1.2 \mathrm{moll}^{-1}\right)$ was carefully placed at $-78^{\circ} \mathrm{C}$ on the monomer solution as a separate liquid phase. The sealed tube was immediately set in the probe of the spectrometer at $-78^{\circ} \mathrm{C}$ so that the butyllithium solution layer was located just at the centre of the irradiation coil. The Fourier-transform NMR spectra of the butyllithium solution were measured at $-78^{\circ} \mathrm{C}$ without spinning the sample tube, and the concentration of butyllithium was followed with time by the intensity measurement of the signal from the protons of methylene group adjacent to the lithium atom, which appeared $3.03 \mathrm{ppm}$ upfield from the resonance of the remaining methyl protons in the solvent toluene- $d_{8}$.

In the polymerization of perdeuterated methyl methacrylate, the filtrate and the washings were combined and evaporated to dryness under reduced pressure. The residue was redissolved in benzene and a small amount of insoluble material was removed by filtration. The methanol-soluble oligomer was recovered from the benzene solution by freezedrying technique. Polymerization was also done by the ordinary method in a glass ampoule under dry nitrogen. The polymer and oligomer were isolated in a way similar to that of the slow growth polymerization.

The isotactic polymer of methyl methacrylate with a deuterated methoxyl group was prepared in the polymerization in toluene by phenylmagnesium bromide at $0^{\circ} \mathrm{C}$. The triad tacticity of the polymer is as follows: I, $96 \% ; \mathrm{H}, 4 \% ; \mathrm{S}, 0 \%$. The monomer was obtained from methacryloyl chloride and perdeuterated methanol at room temperature in ether in the presence of triethylamine.

The isotactic $\left(M_{n}=2657, \mathrm{I}, 85 \% ; \mathrm{H}, 8 \% ; \mathrm{S}, 7 \%\right)$ and syndiotactic $\left(M_{n}=2576, \mathrm{I}, 3 \% ; \mathrm{H}, 10 \% ; \mathrm{S}, 87 \%\right)$ oligomers of methyl methacrylate were obtained as the methanol-soluble fractions of the products formed in the polymerizations of this monomer in toluene by phenylmagnesium bromide at $0^{\circ} \mathrm{C}$ and by bis(pentamethylenimino)-magnesium at $-78^{\circ} \mathrm{C}$, respectively.

The triad tacticity of poly(methyl methacrylate) was determined from the $\alpha$-methyl proton resonances in the ${ }^{1} \mathrm{H}$ NMR spectrum measured in chloroform- $d_{1}$ at $55^{\circ} \mathrm{C}$. The spectrum was taken on a JNM-MH-100 (JEOL) spectrometer at $100 \mathrm{MHz}$, using tetramethylsilane as the internal standard.

The diffusion of oligo(methyl methacrylate) through the solution of isotactic poly(methyl methacrylate) with a deuterated methoxy group in toluene was investigated by using a Fourier- 
Table I. Slow growth polymerization of methyl methacrylate in toluene by butyllithium at $-78^{\circ} \mathrm{C}$ for $24 \mathrm{~h}^{\mathrm{a}}$

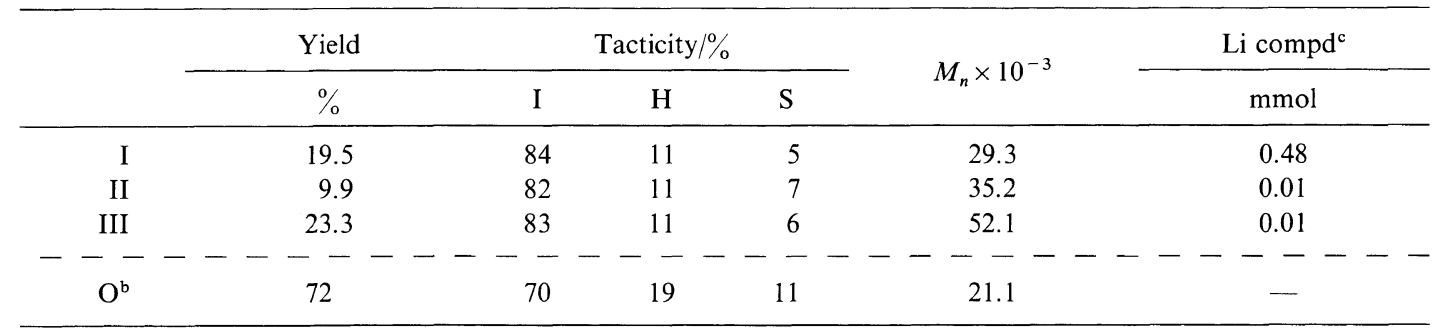

a Monomer, $10 \mathrm{mmol}$; butyllithium, $0.5 \mathrm{mmol}$; toluene, $10 \mathrm{ml}$.

b Polymerization by ordinary method.

c Amount of lithium compound contained in each layer after the polymerization.

transform NMR spectrum at $27^{\circ} \mathrm{C}$. In a $5 \mathrm{~mm}$ NMR sample tube was placed a $0.29 \mathrm{ml}$ solution of the poly(methyl methacrylate- $\left.d_{3}\right)$ in toluene- $d_{8}$ $\left(0.05 \mathrm{~g} \mathrm{ml}^{-1}\right)$, and on this a $0.1 \mathrm{ml}$ solution of isotactic or syndiotactic oligo (methyl methacrylate) in a mixture of toluene and toluene- $d_{8}\left(0.05 \mathrm{~g} \mathrm{ml}^{-1}\right)$ was carefully added. The tube was transferred to the probe of the NMR spectrometer so that the halfheight position of the polymer solution was located at the center of the coil. The spectra of the polymer solution were measured without spinning the sample tube. The amount of the oligomer diffused into the polymer solution was determined by the intensity measurement of the signal of methoxy methyl protons in the oligomer. The degree of diffusion is represented by the proportion in percent of the intensity of the methoxy methyl resonance to that of the completely mixed solutions of oligomer and polymer in the tube.

The number-average molecular weights of polymer and oligomer were measured by a Hitachi 117 vapor-pressure osmometer in benzene at $42^{\circ} \mathrm{C}$.

\section{RESULTS AND DISCUSSION}

Slow growth polymerization of methyl methacrylate was carried out in toluene at $-78^{\circ} \mathrm{C}$ by butyllithium for $24 \mathrm{~h} . *$ The results are shown in Table I together with the results of the polymerization by the ordinary method. The

* In our previous communication, ${ }^{1}$ the polymerization was carried out for $429 \mathrm{~h}$ and the reaction mixture was sliced into four portions instead of three after the polymerization. However, the results were substantially the same as those obtained in this work. isotacticity and the molecular weight of the polymer prepared by the slow growth method were much higher than those of the polymer obtained by the ordinary method. The molecular weight depended on the position in the reaction mixture, increasing from top to bottom.

The amount of lithium compound existing in each portion of the reaction mixture after the polymerization was determined by acid-titration using methyl orange as an indicator. The results indicate that the lithium compound originated from the initiator remained mostly in the upper portion even after the polymerization proceeded over $50 \%$ (Table I). The change in the concentration of butyllithium in the layer of the initiator solution was followed during the polymerization in an NMR sample tube by the Fourier-transform NMR method mentioned in the experimental section. The results

Table II. Concentration of remaining butyllithium in the layer of initiator solution in the course of the slow growth polymerization of methyl methacrylate in toluene at $-78^{\circ} \mathrm{C}$

\begin{tabular}{cc}
\hline Time & $\begin{array}{c}\text { Concentration of } \\
\text { butyllithium } / \%\end{array}$ \\
\hline $\mathrm{h}$ & 100 \\
0 & 100 \\
0.28 & 97 \\
0.55 & 93 \\
0.78 & 93 \\
1.15 & 93 \\
2.23 & 91 \\
24.0 & \\
\hline
\end{tabular}


Table III. Effect of polymerization time on the slow growth polymerization of methyl methacrylate by butyllithium in toluene at $-78^{\circ} \mathrm{C}^{\mathrm{a}}$

\begin{tabular}{|c|c|c|c|c|c|c|}
\hline \multirow{2}{*}{$\begin{array}{c}\text { Polymerization time } \\
\mathrm{h}\end{array}$} & \multirow{2}{*}{$\begin{array}{l}\text { Yield } \\
\%\end{array}$} & \multirow{2}{*}{$M_{n} \times 10^{-3}$} & \multirow{2}{*}{$\begin{array}{l}\text { Amount of polymer } \\
\text { chain } \times 10^{3} / \mathrm{mmol}\end{array}$} & \multicolumn{3}{|c|}{ Tacticity $/ \%$} \\
\hline & & & & I & $\mathrm{H}$ & $\mathrm{S}$ \\
\hline 1 & 1.6 & 18.7 & 0.9 & 74 & 17 & 9 \\
\hline 2 & 3.6 & 23.2 & 1.5 & 76 & 16 & 8 \\
\hline 4 & 16.9 & 43.1 & 3.9 & 83 & 12 & 5 \\
\hline 24 & 23.3 & 52.1 & 4.5 & 83 & 11 & 6 \\
\hline 168 & 32.7 & 73.5 & 4.5 & 85 & 11 & 4 \\
\hline
\end{tabular}

a Monomer, $10 \mathrm{mmol}$; butyllithium, $0.5 \mathrm{mmol}$; toluene, $10 \mathrm{ml}$. The data are concerned with the polymer formed in the third portion (bottom) of the reaction mixture.

are given in Table II. Over $90 \%$ of the butyllithium used was found to exist unchanged in the layer even after $24 \mathrm{~h}$, during which over a half of the monomer changed into the polymer. These facts indicate that only a small fraction of the added butyllithium was used for the initiation of methyl methacrylate, resulting in the formation of a polymer of higher molecular weight than that of the polymer prepared by the ordinary method.

The effect of the polymerization time is shown in Table III. The data in the Table are concerned with the polymers isolated from the third portions of the reaction masses, whose weights were almost the same. The yield and molecular weight of the polymer increased with increasing polymerization time. The amount in mmoles of the polymer molecule formed, calculated from the yield and the molecular weight of the polymer, also increased with increasing polymerization time, especially in the initial stage of the polymerization. The isotacticity of the polymer increased with an increase in the polymerization time. A similar tendency was also observed in ordinary polymerization. ${ }^{6}$

From the results mentioned above, the following conclusions can be drawn on the slow growth polymerization by butyllithium in toluene: The initiation occurs at the interface between the initiator and monomer solutions and the active species thus formed gradually diffuse from top to bottom, growing into the polymer chain of higher molecular weight. As a result of this, the molecular weight of the formed polymer increased from top to bottom. Though the apparent rate of the initiation is slow, it is continuous and the number of polymer chains in the reaction mixture increases during the polymerization.

One of the characteristic features of this slow growth polymerization is the formation of a polymer of higher isotacticity than that of the polymer prepared by ordinary polymerization. In order to clarify the mechanism for this increase in the isotacticity, the rate of diffusion of the isotactic or syndiotactic oligomer of methyl methacrylate into the solution of isotactic poly(methyl methacrylate) in toluene was measured by using the Fouriertransform NMR. This method was described in the experimental section and the results are shown in Figure 2. The isotactic oligomer slowly diffused through the solution of the isotactic polymer. The syndiotactic oligomer did not diffuse into the polymer solution, although the toluene molecules added to the oligomer solution diffused gradually. The formation of a stereocomplex may occur between the isotactic polymer and the syndiotactic oligomer at the interface, and this may prevent the diffusion of the oligomer molecules.

In our previous paper ${ }^{7}$ it was shown that there exist isotactic and syndiotactic active species in the polymerization of ethyl methacrylate by butyllithium in toluene. It may be true in the polymerization of methyl methacrylate. The increase of the isotacticity in the slow growth polymerization may thus be explained in the following way. In the slow growth polymerization in toluene, the isotactic and syndiotactic active species simultaneously form at the interface between the solutions of initiator and monomer, where the isotactic species are predominant in a nonpolar solvent such as toluene. The diffusion of syndiotactic species into the reaction mixture is interfered with by a larger amount of the 
Table V. Slow growth polymerization of perdeuterated methyl methacrylate in toluene by butyllithium at $-78^{\circ} \mathrm{C}$ for $24 \mathrm{~h}^{\mathrm{a}}$

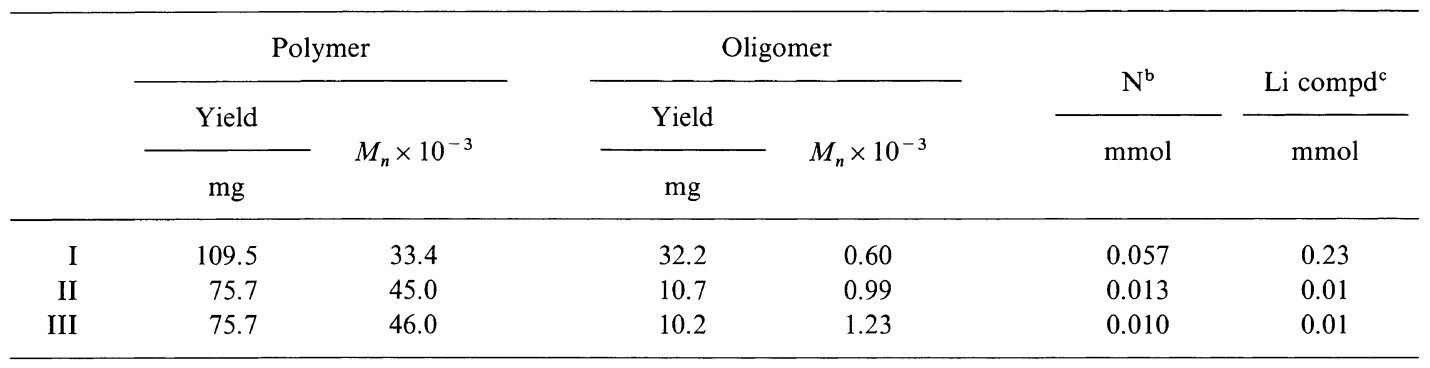

a Monomer, $4.97 \mathrm{mmol}$; butyllithium, $0.25 \mathrm{mmol}$; toluene, $10 \mathrm{ml}$.

b Total amounts in mmol of polymer and oligomer molecules formed during the polymerization.

c Amount of lithium compound in the reaction mixture after the polymerization.

corresponding portion. Both were in good agreement with each other, except for the top portion (I) where unreacted butyllithium also existed (Table V).

The results mentioned here suggest that in the slow growth polymerization, the extent of the carbonyl attack by butyllithium is lower than in the ordinary polymerization and the lithium methoxide formed does not enter into the reaction mixture, probably owing to a complex formation with the remaining butyllithium so that the propagation reaction occurs almost free from the methoxide to enhance the formation of the highly isotactic polymer.

The slow growth polymerization of methyl methacrylate was carried out in toluene with 1,1diphenylhexyllithium at $-78^{\circ} \mathrm{C}$. In this case, the polymerization was initiated by adding slowly the monomer solution onto the initiator solution because of the higher density of the latter solution. The deep red color of the initiator remained almost unchanged during the polymerization, and after $24 \mathrm{~h}$, the polymer was obtained only in the bottom portion (III). The results are shown in Table VI, together with those of ordinary polymerization. The polymer prepared by the slow growth method was similar in stereoregularity to those prepared by the ordinary method and also by the slow growth polymerization initiated with butyllithium in toluene. In the polymerization by 1,1diphenylhexyllithium in toluene, the amount of lithium methoxide formed was found to be much less than with butyllithium. ${ }^{8}$ The less lithium methoxide and, accordingly, the low level of the syndiotactic active species are the reasons as to why the increase in the isotacticity cannot be observed in the slow
Table VI. Slow growth polymerization of methyl methacrylate in toluene by 1,1-diphenylhexyllithium at $-78^{\circ} \mathrm{C}^{\mathrm{a}}$

\begin{tabular}{rcccccc}
\hline & \multicolumn{2}{c}{ Yield } & & \multicolumn{4}{c}{ Tacticity/\% } \\
\cline { 2 - 3 } \cline { 5 - 7 } & $\%$ & & I & H & $\mathrm{S}$ \\
\hline I & 0.0 & & - & - & - \\
II & 19.4 & & 86 & 11 & 3 \\
\hline$O^{\text {III }}$ & 94.1 & & 83 & 13 & 4 \\
\hline
\end{tabular}

a Monomer, $10 \mathrm{mmol}$; initiator, $0.5 \mathrm{mmol}$; toluene, $10 \mathrm{ml}$.

b Polymerization by ordinary method.

growth polymerization by 1,1-diphenylhexyllithium.

In conclusion, the formation of a stereocomplex between isotactic and syndiotactic active species, together with the reduced amount of lithium methoxide in the reaction mixture, may contribute to the increase in the isotacticity in the slow growth polymerization of methyl methacrylate in toluene by butyllithium. It is rather difficult at the present to decide which contributes predominately. It may depend on the structure of the monomer and an extensive study is now under way on the slow growth polymerization of alkyl methacrylates other than methyl methacrylate.

Acknowledgment. A part of this work was supported by a grant-in-aid for scientific research from the Ministry of Education, Japan. The authors are grateful to $\mathrm{Mr}$. Takatoshi Ochi for the preparation of perdeuterated methyl methacrylate and to Mrs. F. Yano for her clerical assistance in 
preparing this manuscript.

\section{REFERENCES}

1. H. Yuki, K. Hatada, and S. Kokan, Polym. J., 5, 329 (1973).

2. K. Hatada, S. Kokan, and H. Yuki, J. Polym. Sci., Polym. Lett. Ed., 13, 721 (1975).

3. K. Hatada, M. Furomoto, and H. Yuki, Makromol. Chem., 179, 1107 (1978).

4. K. Hatada, T. Kitayama, K. Fujikawa, K. Ohta, and
H. Yuki, Polym. Bull., 1, 103 (1978).

5. J. W. C. Crawford, Br. Patent 405699 (1934).

6. D. M. Wiles and S. Bywater, Polymer, 3, 175 (1962).

7. K. Hatada, T. Kitayama, H. Sugino, Y. Umemura, M. Furomoto, and H. Yuki, "Advances in the Preparation and Properties of Stereoregular Polymers," NATO Advanced Study Institute, October, 1978, Tirrenia, Pisa, Italy; Polym. J., 11, 989 (1979).

8. D. M. Wiles and S. Bywater, Trans. Faraday Soc., 61, 150 (1965). 